\title{
X-ray photoemission electron microscopy investigation of magnetic thin film antidot arrays
}

\author{
L. J. Heyderman ${ }^{\text {a) }}$ \\ Laboratory for Micro- and Nanotechnology, Paul Scherrer Institut, CH-5232 Villigen PSI, Switzerland \\ F. Nolting and C. Quitmann \\ Swiss Light Source, Paul Scherrer Institut, CH-5232 Villigen PSI, Switzerland
}

(Received 2 May 2003; accepted 1 July 2003)

\begin{abstract}
Periodic square arrays of antidots in $10 \mathrm{~nm}$-thick cobalt films have been investigated with antidot periods, $p$, ranging from $2 \mu \mathrm{m}$ down to $200 \mathrm{~nm}$ and various ratios of antidot size to antidot separation, $w / d$. For $p=2 \mu \mathrm{m}$, the extent of modification of the thin film magnetic domain structure increases with increasing $w / d$, forming domains pinned diagonally between antidots for $w / d$ $\geqslant 0.2$ and resulting in a two-dimensional periodic checked domain contrast commensurate with the antidot lattice for $w / d \geqslant 0.9$. As $p$ is decreased while maintaining $d \approx w$, we observe a dramatic change in the magnetic domain configuration at $p=400 \mathrm{~nm}$ resulting in chains of magnetic domains running parallel to the intrinsic hard axis and with lengths corresponding to a multiple of the antidot period. (C) 2003 American Institute of Physics. [DOI: 10.1063/1.1605804]
\end{abstract}

Magnetic thin films with periodic arrays of nonmagnetic inclusions referred to as antidots have been suggested as a possible candidate for ultrahigh density storage. ${ }^{1}$ Around the antidots, a stable domain configuration is formed which is a result of the interplay of the intrinsic uniaxial anisotropy of the magnetic thin film and the demagnetizing fields associated with the antidots which tend to align the magnetization parallel to the edge of the antidot. The resulting domain configuration contains regions with remanent magnetization parallel to the intrinsic hard axis of the magnetic thin film (the white regions in Fig. 1), which can be used to store bits of information ${ }^{1,2}$ and it has been predicted that in such systems areal densities of $750 \mathrm{Gbits} / \mathrm{in}^{2}$ can be achieved. ${ }^{3}$ Antidot arrays are also of interest because they significantly modify the switching characteristics in magnetic thin films and it is possible to tailor both the switching fields and magnetoresistance behavior by changing the size, separation, and the lattice symmetry and orientation of the antidots. ${ }^{4-7}$

In the present study, we have investigated the as-grown magnetic domain configurations in square lattice antidot systems for a wide range of periods, $p=d+w$, from $2 \mu \mathrm{m}$ down to $200 \mathrm{~nm}$ and various $w / d$ with $p=2 \mu \mathrm{m}$ (see Fig. 1 for antidot geometry). These periods and antidot sizes go far below previously reported magnetic domain observations in antidot arrays, where the lowest dimensions were $p \approx 1 \mu \mathrm{m}$ and $d \approx 500 \mathrm{~nm}$. For magnetic domain observations, we used $\mathrm{x}$-ray photoemission electron microscopy (PEEM). This observation technique has a significant advantage over magnetic force microscopy studies ${ }^{8,9}$ being nonintrusive and providing direct imaging of the magnetization orientations. In contrast to Lorentz microscopy, ${ }^{10}$ no special preparation of the sample substrate is required.

We employed electron beam lithography to fabricate the antidot arrays using a lift-off process to transfer the antidot pattern written in a polymethylmethacrylate resist on a $\mathrm{Si}$

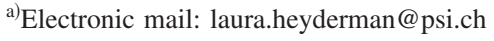

(001) substrate into $10 \mathrm{~nm}$-thick polycrystalline cobalt films. The films have a $1 \mathrm{~nm}$-thick aluminum capping layer to prevent oxidation of the magnetic film and are deposited by dc-magnetron sputtering. We produced various antidot separations with values ranging from just below $p$ down to $120 \mathrm{~nm}$, so providing a series of $w / d$. A scanning electron microscopy (SEM) image of the smallest period antidot array, $p=200 \mathrm{~nm}$, is shown in the inset of Fig. 3(b). Due to the limited resolution of fabrication process, the antidots change in geometry from square to round holes when $w \leqslant 150 \mathrm{~nm}$. Hysteresis loop measurements were carried out on a separate reference sample with a continuous cobalt film of thickness $10 \mathrm{~nm}$, grown using the same deposition system. The continuous film displayed a uniaxial anisotropy, with an anisotropy field value of about 50 Oe and an easy axis coercivity of about 25 Oe. The uniaxial anisotropy is likely to be due to a small field (20-30 Oe) present during sputter deposition.

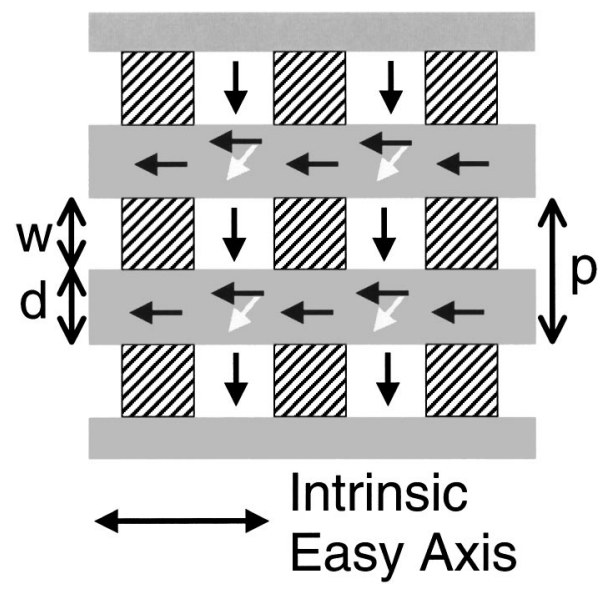

FIG. 1. Schematic diagram of antidot array with ideal remanent state for storage; the nonmagnetic regions are hatched, $w$ is the antidot size, $d$ is the antidot separation, and $p=d+w$ is the antidot period. In the gray/white regions, the magnetization (black arrows) is parallel to the intrinsic easy/hard axis. Detailed micromagnetic calculations predict a $45^{\circ}$ rotation of the magnetization as indicated by the white arrows. 


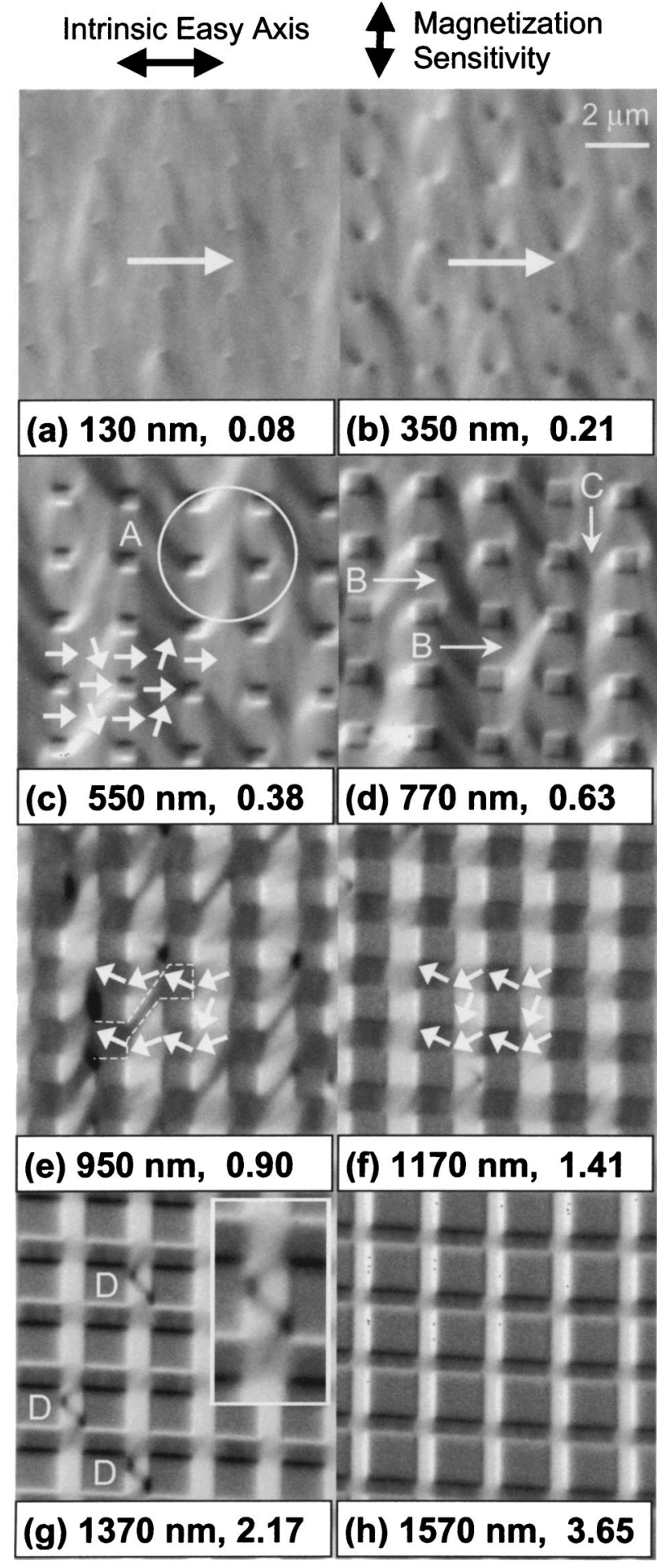

FIG. 2. Magnetic domain configurations associated with antidot arrays with period, $p=d+w=2 \mu \mathrm{m}$. The antidot size $w$ and ratio $w / d$, where $d$ is the antidot separation, are given in the box for each image.

The imaging was carried out at the SIM beamline ${ }^{11}$ at the Swiss Light Source, Paul Scherrer Institut, with an Elmitec PEEM. Employing x-ray magnetic circular dichroism (XMCD), the magnetic domains are imaged by tuning the $\mathrm{x}$-ray energy to the Co $L$ edge. Dividing two images taken with left and right circular polarized light leads to an XMCD image where the intensity is a measure of the angle between the circular x-ray polarization vector, labeled as magnetization sensitivity direction in the figures, and the magnetization in the domains. ${ }^{12}$ Ferromagnetic domains with magnetization parallel or antiparallel to the polarization vector appear black or white in the XMCD image and oppositely magnetized domains with magnetization perpendicular to the polarization vector will both have the same gray contrast.

The domain structures for $p=2 \mu \mathrm{m}$ and $w / d$ ranging from 0.08 to 3.65 are shown in Fig. 2. For very small antidot sizes, i.e., $w / d \leqslant 0.21$, we observe a slight modification to the magnetization orientation which is predominantly parallel to the easy axis. Low contrast "whiskers" form at the edges of the antidots to reduce the demagnetization energy associated with the dots and are comparable to the spike domains calculated from micromagnetic theory. ${ }^{13,14}$ The modification of the magnetization by the antidots increases as the antidot size increases. For $w / d=0.38$ and 0.63 , while some whisker domains are still present, for example within the circle at $\mathrm{A}$ in Fig. 2(c), the demagnetizing field associated with the antidots is large enough to induce the formation of domains which run diagonally between antidots. The diagonal domains are either black or white as both "up" and "down" orientations of the magnetization are equally favorable in the as-grown magnetic state, and they resemble the diagonal domains calculated by Refs. 14 and 15. It is also interesting to note that in a given column between antidots, the magnetization in the adjacent diagonal domains tends to be parallel, i.e., all black or all white. However, there are disruptions to this order and of particular interest in Fig. 2(d) are the regions where two diagonal domains cross each other, for example the two crossing points indicated by $\mathrm{B}$, and also where two diagonal half-domains of opposite contrast meet, for example in the column between antidots indicated by $\mathrm{C}$. These diagonal half-domains are similar to the whisker domains in Fig. 2(c) but have a stronger, more defined contrast.

When $d$ approaches $w$ [Figs. 2(e) and 2(f)], we observe a change in the magnetic domain configuration to a checked domain pattern with three domain contrasts, which is periodic in two dimensions and commensurate with the antidot array. This is the domain configuration shown in Fig. 1. From the simple schematic of the domain magnetizations in Fig. 1 (black arrows), we would expect to see only two domain contrasts. However, detailed micromagnetic theory does indeed predict three domain contrasts where the magnetization in the regions not directly adjacent to the antidots is rotated by $45^{\circ}$ (indicated by the white arrows in Fig. 1). ${ }^{14,15}$ Careful evaluation of the magnetic contrast indicates that there are slight deviations of the magnetization from the intrinsic easy and hard axes as given by the white arrows in Figs. 2(e) and 2(f). Such deviations can be a result of a slight misalignment of the local easy axis with the antidot array. ${ }^{10}$

When $w / d$ is slightly less than 1 [Fig. 2(e)], some of the easy axis domains are joined by narrow diagonal domains. The number of such "defect" domains decreases as $w / d$ increases and are not seen for $w / d=3.65$ [Fig. 2(h)]. This improvement is likely to be due to the fact that the exchange and demagnetizing energies do not favor such changes in the magnetization orientation when the lateral dimensions of the magnetic material between the antidots is decreased, in this case to $430 \mathrm{~nm}$. Note also the interesting but reproducible $360^{\circ}$ wall-type irregularities marked by D in Fig. 2(g) and shown enlarged in the inset.

We now concentrate on the antidot arrays with $d \approx w$ and 


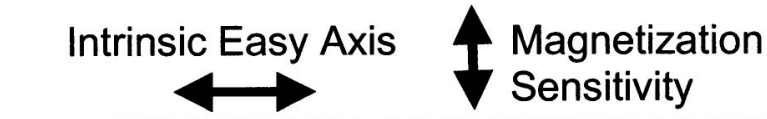

(a) p: $400 \mathrm{~nm}, \mathrm{w:} 200 \mathrm{~nm}$

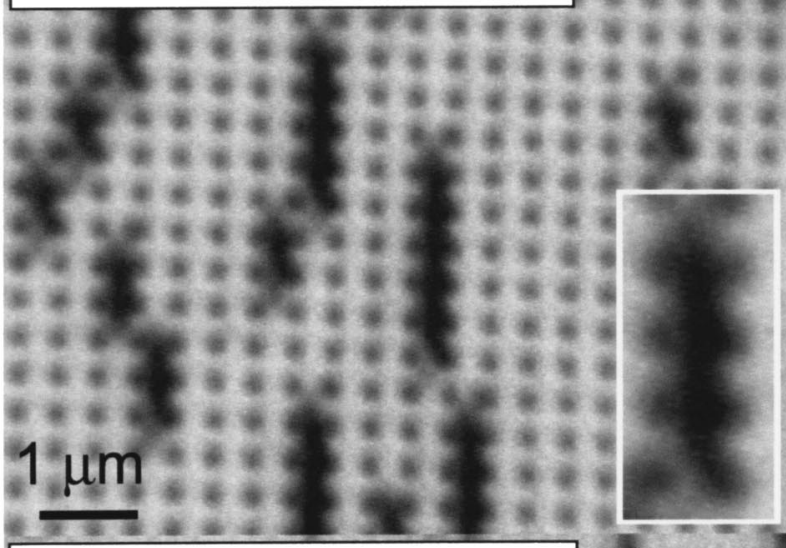

(b) p: $200 \mathrm{~nm}, \mathrm{w}: 80 \mathrm{~nm}$

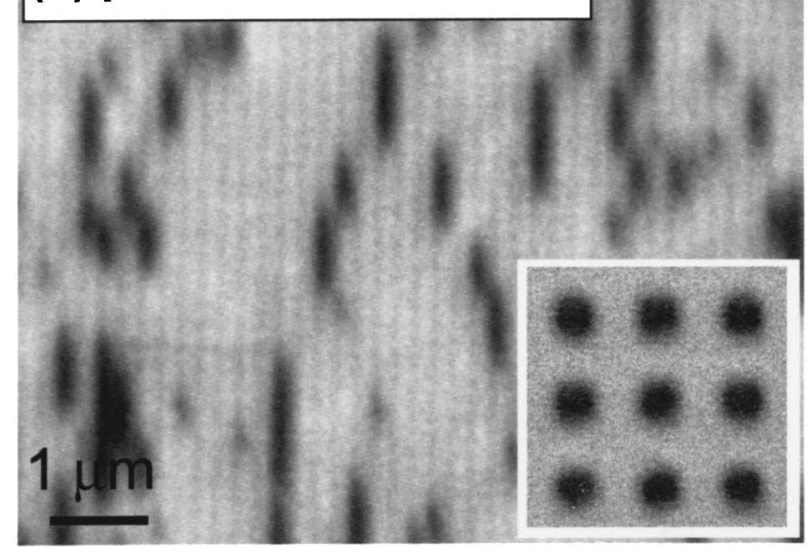

FIG. 3. Magnetic domain configurations associated with antidot arrays with $d \approx w$. The period, $p$, and antidot size, $w$, are given in the box for each image. The inset in (a) is a close up of one of the magnetic domain chains and the inset in (b) is an SEM image of the $200 \mathrm{~nm}$ period antidot array.

observe what happens in the as-grown magnetic state as the period decreases. Checked domains with two-dimensional periodicity are also seen for a period of $1 \mu \mathrm{m}$. However, at a period of $400 \mathrm{~nm}$ [Fig. 3(a)], we observe a completely different magnetic configuration: chains of domains running parallel to the intrinsic hard axis, which have different lengths corresponding to multiples of the antidot period. Surprisingly, here the magnetization in the dark and light regions is approximately parallel to the intrinsic hard axis and such magnetic domain chains are observed down to an antidot period of $200 \mathrm{~nm}$ [Fig. 3(b)], which is at the current resolution limit of $150 \mathrm{~nm}$ of the PEEM microscope. On closer inspection, see inset in Fig. 3(a), the dark contrast appears to be a result of chains of domains which diagonally join antidots. This is reminiscent of the diagonal domains in Figs. 2(c) and 2(d), and we believe that similar magnetic energy principles govern their formation. In addition, this change in domain configuration may be related to the sudden drop in the signal-to-noise ratio curve calculated in Ref. 14. If the final length of the chains is not defined by the presence of defects, it may be possible to switch individual adjacent regions to change the chain length at will, so providing a method of information storage with the recording track parallel to the chain.

In conclusion, we have investigated the modification of the as-grown magnetization distribution in thin magnetic films containing antidot arrays with a wide range of $p$ and $d / w$ using X-ray PEEM. The observed magnetic domain configurations for $p \geqslant 1 \mu \mathrm{m}$ substantiate the micromagnetic calculations of Refs. 13-15. A dramatic change in the magnetic domain configuration at $p=400 \mathrm{~nm}$ occurred resulting in chains of coupled magnetic domains running parallel to the intrinsic hard axis. The appearance of this domain configuration has important implications for tailoring of magnetic thin film properties and storage of magnetic information.

The authors would like to thank Luis López-Díaz of Salamanca University for valuable advice and discussions, and at the Paul Scherrer Institut: Michael Horisberger for the sputter deposition, Oksana Zaharko for hysteresis loop measurements, and Harun Solak and Christian David for their support with electron beam lithography. Part of this work was performed at the SLS, Paul Scherrer Institut, Villigen, Switzerland.

${ }^{1}$ R. P. Cowburn, A. O. Adeyeye, and J. A. C. Bland, J. Magn. Magn. Mater. 173, 193 (1997).

${ }^{2}$ R. P. Cowburn, A. O. Adeyeye, and J. A. C. Bland, Appl. Phys. Lett. 70, 2309 (1997).

${ }^{3}$ L. Torres, L. Lopez-Diaz, O. Alejos, and J. Iniguez, Physica B 275, 59 (2000).

${ }^{4}$ A. O. Adeyeye, J. A. C. Bland, and C. Daboo, Appl. Phys. Lett. 70, 3164 (1997).

${ }^{5}$ Z. L. Xiao, Y. C. Han, U. Welp, H. H. Wang, V. K. Vlasko-Vlasov, W. K. Kwok, D. J. Miller, J. M. Hiller, R. E. Cook, G. A. Willing, and G. W. Crabtree, Appl. Phys. Lett. 81, 2869 (2002).

${ }^{6}$ M. B. A. Jalil, S. L. A. Phoa, S. L. Tan, and A. O. Adeyeye, IEEE Trans. Magn. 38, 2556 (2002).

${ }^{7}$ I. Ruiz-Feal, L. Lopez-Diaz, A. Hirohata, J. Rothman, C. M. Guertler, J. A. C. Bland, L. M. Garcia, J. M. Torres, J. Bartolome, F. Bartolome, M. Natali, D. Decanini, and Y. Chen, J. Magn. Magn. Mater. 242-245, 597 (2002).

${ }^{8}$ C. T. Yu, H. Jiang, L. Shen, P. J. Flanders, and G. J. Mankey, J. Appl. Phys. 87, 6322 (2000).

${ }^{9}$ P. Vavassori, G. Gubbiotti, G. Zangari, C. T. Yu, H. Yin, H. Jiang, and G. J. Mankey, J. Appl. Phys. 91, 7992 (2002).

${ }^{10}$ A. Y. Toporov, R. M. Langford, and A. K. Petford-Long, Appl. Phys. Lett. 77, 3063 (2000).

${ }^{11}$ C. Quitmann, U. Flechsig, L. Patthey, T. Schmidt, G. Ingold, M. Howells, M. Janousch, and R. Abela, Surf. Sci. 480, 173 (2001).

${ }^{12}$ A. Scholl, H. Ohldag, F. Nolting, J. Stohr, and H. A. Padmore, Rev. Sci. Instrum. 73, 1362 (2002).

${ }^{13}$ L. Lopez-Diaz, L. Torres, and J. I. Iniguez, J. Magn. Magn. Mater. 196197, 805 (1999).

${ }^{14}$ L. Torres, L. Lopez-Diaz, and J. Iniguez, Appl. Phys. Lett. 73, 3766 (1998).

${ }^{15}$ L. Torres, L. Lopez-Diaz, O. Alejos, and J. Iniguez, J. Appl. Phys. 85, 6208 (1999). 